\title{
Digital Morphogenesis: A Synthetic Approach to Generate Architectural Elaborate Components
}

\author{
Jiarui Liu ${ }^{1,2}$ a,b, Zhiyu Guan ${ }^{3, c}$, Xin Chen ${ }^{1, d}$, Chunyi Xu u $^{1, e}$ \\ ${ }^{1}$ Shenyang Jianzhu University, China \\ ${ }^{2}$ Shenyang Ouya Civil Engineering Design Co.,Ltd., China \\ ${ }^{3}$ Zhongtian Construction Group Co.,Ltd. Northeast Company, China \\ aJerome.liujiarui@outlook.com, buczlj28@ucl.ac.uk, clividio@126.com, \\ 'chenxin_zoe@163.com, ${ }^{\mathrm{e}}$ manway1029@sina.com
}

\begin{abstract}
Keywords: Architectural Design; Digital Morphogenesis; Elaborate Components; Generated Approach; Digital Prefabrication

Abstract. The convoluted nature of contemporary architectural design stands as an innovation trend. However, this tendency could hardly be supported by traditional computational assist design technique. The purpose of this research is to present digital morphogenesis technologies increase the accessible of generation architectural elaborate components. The application of digital morphogenesis on the design of architectural elaborate components enabled the emergence of convoluted nature of contemporary architectural design. It is believed that digital morphogenesis could provide the methods from the term of design, analysis and manufacture. This paper suggests that digital morphogenesis could deliver an available process for creative architectural elaborate components.
\end{abstract}

\section{Introduction}

Digital morphogenesis is prominent promoting the development of contemporary design. It is a novel method and tool to create complexity morphology and process, which lead to an unconventional aesthetic and technical complexity. This approach depended on logic understood through the esoteric study of the natural morphogenetic rules which creative the structural nature processes [1]. Neil Leach [2] traces digital morphogenesis design presents the potential of the computational realm through the opening up the design fields of possibilities. Also, this has been explored by some avant-garde architects, such as Achim Menge and Frei Otto, in diverse ways. Besides, Michael Weinstock [3] claims that produce emergent morphogenesis and behavior is the origins of the notion, which is obtained by mathematical basic of processes. The utilization of mathematical models can simulate the calculation of the pattern or structure formation process both in natural conditions and computational environments. Digital morphogenetic methods are evolutionary since incorporate iterations of physical modelling. The emergence of digital morphogenesis due to the booming of computer science, which have already influence the manufacture of elaborate architectural components. Traditionally, it is nearly impossible to meet the requirement of an unusual morphogenesis[4], which is limited to the design tools and manufacture process. However, the soaring development of computational technology promoted the application of digital morphogenesis on the domain of elaborate prefabrication in last decade, the process of computational technology has evolved into design participants from a simple assist drawing tools.

The purpose of this paper is to present that what digital morphogenesis could deliver for the design and fabrication of architectural elaborate components. The study investigated from elaborate geometry generation, computational analysis and digital fabrication. Specifically, the study elaborated: design algorithm to easy the complexity morphology; using computational simulation assist design and utilized the digital method to realize the unconventional morphogenesis. 


\section{Algorithmically generated geometry}

Digital morphogenesis delivers an opportunity for architects that converting the end state of what they are designing into unimaginable shapes. Architects did design using algorithm program, such as processing and grasshopper, to define the generation rule of geometry without pen or by mouse in a $C A D$ program. Geometry could be designed with a complexity and richness which would create highly differentiated and spatially complex architecture.

Architects could sculpt the form, even though, they would not imagine the end state at the beginning of the project. Just as what Hansmeyer[5] did in the platonic solids project(Fig. 1). Those abstracted the process of creation, morphogenesis, is asymmetric cell division. Designer exerted control over the process that generated the form instead of design the form. The morphogenesis of resulting presented an unusual aesthetic and technical complexity that likely defies intend to reductionism. In esoteric realm, if the designer makes a small change to the parameter such as folding ratios, it would lead to the shape changes correspondingly.

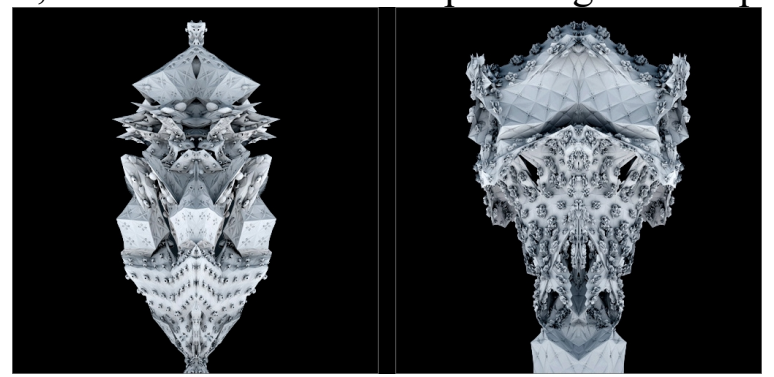

Fig. 1, Platonic solids project

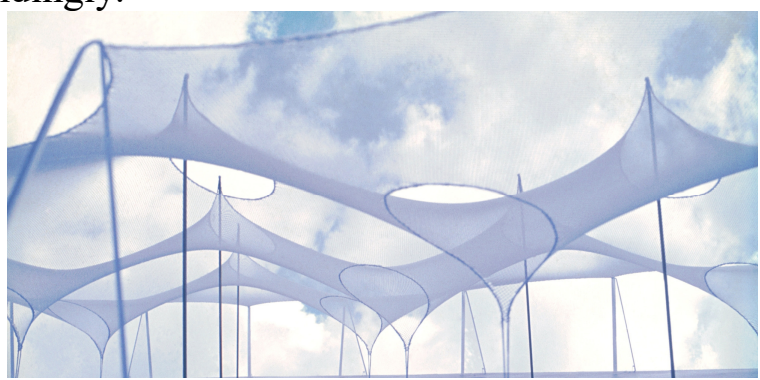

Fig. 2, Frei Otto's "form-finding"

Moreover, Architects could define forms and methods that borrow nature's processes, and then code them as algorithms to explore unimaginable shapes. Frei Otto, a highly renowned architect, who obsessed of research on nature and computation, was one of the first to apply "form-finding" technique to the architectural design. Amounts of elaborate structures began its generate logic with "form-finding" technique[6]. Those buildings present a clear and logical "form-finding" process(Fig2). This method created logic through the technology of digital model simulation to find morphogenetic rule from the laws of nature and also find the process of the natural pattern and structure. Since there is not man-made interference during the process, it known as the "Self-formation" procedure.

Thus, developments in digital morphogenesis made architects have access to create elaborate architectural components that is excited new. Designers could define themselves as a facilitator rather than a designer as well as define nature as a rule-maker in the process of algorithmic architecture. Additionally, the utilization of algorithm independent developers from their experience, no bias and no preconceptions. These allows designers to create structures at all scales by developing a process, including those, even have dreamt up.

\section{Analogue and digital analysis}

Phillip Ball[7] points out that mathematics and geometric could describe patterns and forms. It means that digital data could represent that whole of surfaces and details of designs. Computational morphogenesis design focus on the analysis and production of complex shapes and behaviour. It could provide optimal solutions for designers by simulate and analyse digital models with particular conditions, especially in highly sophisticated data analysis. Additionally, Michael Hansmeyer expounds that simulation of morphogenesis generated on computer environment could escape from physical constraints. It means that designer could make any possible tests that they otherwise would not go. For example, surfaces could intersect themselves, and become mini-scale as possible. Even if the forms of these surfaces stretched or tear, it would not be a dilemma. Above all, digital analysis shows the scope of morphogenesis which architects can design. 
Besides, there is a relevant example Eden project(Fig.3) which presents that how concepts from biology and computation help designer radical reduce waste in resource efficiency. Architects devise the most efficient structural scheme, utilization of hexagons and pentagons with the assistance of computational analysis, through abstract morphogenesis from pollen grains and carbon molecules. This structure solution provided an efficient use of steel and top overall performance. Also, it cut down the cost of foundations, due to the utilization of less steel reduced the overall weight in the structure. On the other hand, this form gets extra sunlight into the interior, and it means power-saving in winter because receiving additional external heat. Those are the key to radical increases in resource efficiency.
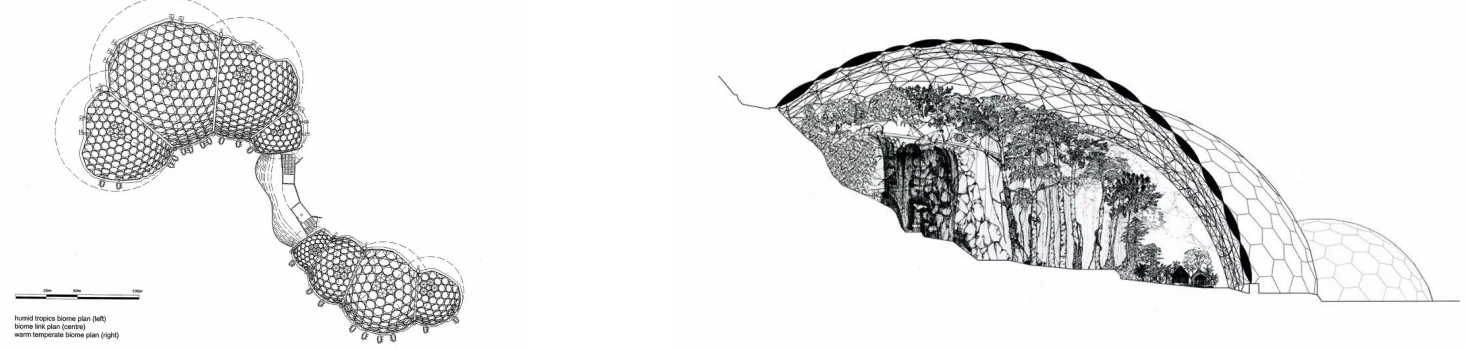

Fig. 3, Eden project

Hence, the computational analysis provides is a useful method of architectural elaborate components design in an efficient way. Architects could test any ideas without considering about cost and realize. Also, designers would sculpt the morphogenesis that is abundance and synergy due to the computational would find the most optimization layout.

\section{Digital materially elaborate fabrication}

Digital morphogenesis could provide complexity machining. It could promote the productive of complexity prefabrication by the computational algorithm. These between material, morphogenesis, structure and fabrication defined through the algorithm that becomes an integral component of the digital design tool. Digital elaborate geometries contain information at very many scales. It would take even years to drew all the drawings, almost impossible for a designer to specify or conceive of. The application of computational technology of morphogenesis fabrication can help architects achieve their proposals, without worried about that complex shapes and structures cannot produce. Computational models would preserve all the information of fabrication, even all of the surface intricacies. These make architects involved in the manufacture of elaborate architectural prefabrication in an efficient way and more widely adopted.
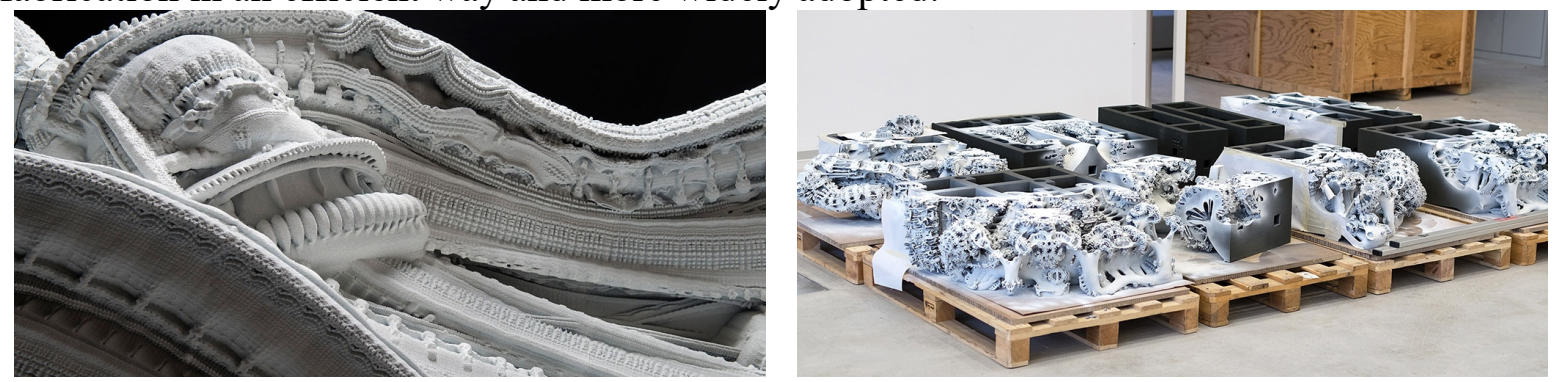

Fig. 4, Arabesque wall project

Architectural elaborate(Fig. 4) components are highly differentiated and spatially complex structure, unlike traditional design, both the overall morphogenesis and the microscopic surface detail is a single process. Some formations might be smaller than the threshold of human visibility. So it demands more about mini-scale fabrication. In the past, ornament and formal expression cease in tiny-scale was a luxury. However, computational technology products could be fabricated at nanoscale components which both in design and manufacture process with the feature of complex morphogenesis, with the help of computational design and digital fabrication. Employing 3D printing 
or industry robot for fabrication of elaborate architectural works herald architects could now achieve their complexity and mini-scale design. Further, an excellent example is the arabesque wall project which present ornamental details at the scale of millimetres. The rhythmic of arabesque wall was produced sophisticated. Interwoven curve exhibit perfect touch and offers new impressions from any perspective. This wall is same as arabesque ornaments, both geometric and mathematical become the compositional principles of it. The primary component is an iterative tiny and division of surfaces. A design process folds a single surface repeatedly produce a structure composed of millions of little facets emerges. There is a dramatic impact on this fabrication because creating such a complexity and richness of detail. Otherwise, it would not be materialized with relative ease.

Therefore, digital materially fabrication provides the future of architectural elaborate design at the nanoscale. This technology not only would be highly efficient but also produces beautiful and organic decorative effects and formal effects. In some case, elaborate architecture could escape the paradigms of rationalization and standardization to produce exquisite detailing and elaborate texture.

\section{Conclusions}

This essay has attempt to evaluate whether initial design strategies and fabrication might be replaced and promoted by computational morphogenesis, especially in the domain of architectural elaborate components. Initially, it expounded that architects develop an algorithm for digital morphogenesis to easy the design process of architectural elaborate components. Further, the computational analysis could provide amounts of tests the elaborate components that might not be available in the physical environment. Last but not the least, the application of digital morphogenesis would make the fabrication of complexity prefabrication, especially sophisticated and complex stuff, is likely to be fabricated.

In conclusion, digital morphogenesis has become a newly arisen method of architectural elaborate components design. This paper proves that various perspectives need be to take into consider. In this essay, it might be true that there are limitations when evaluating the application of digital morphogenesis in the field of elaborate components. Architects will consider more about expand the generated logic and fabrication for the whole building design to fully embrace what digital morphogenesis can provide in nearly future.

\section{Acknowledgement}

This work was financially supported by Ministry of Science and Technology(PRC) 13th five-year plan: Building Technology Basic Theory of Precast Concrete Industrialization (grant number: 2016YFC07014-02) and National Natural Science Foundation of China(grant number: 51408373). We thank our colleagues from Shenyang Jianzhu University who provided insight and expertise that greatly assisted the research, although they may not agree with all of the interpretations of this paper.

\section{References}

[1] Hensel, Michael; Menges, Achim; Weinstock, Michael (eds). Emergence: Morphogenetic Design Strategies. John Wiley.(2004)

[2] Leach, N. Digital morphogenesis. Architectural Design, 79(1), 32-37.(2009)

[3] Weinstock, M. Morphogenesis and the Mathematics of Emergence. Architectural design, (3), 10-17.(2004)

[4] Pawlyn, M. Biomimicry in Architecture (Vol. 15). Riba Publishing.(2011) 
[5] Hansmeyer, M. Subdivision beyond smoothness. In Proceedings of the Sixth International conference on Computational Aesthetics in Graphics, Visualization and Imaging (pp. 75-81). Euro Graphics Association.(2010)

[6] Drew, P. Frei Otto: form and structure. Westview Press.(1976)

[7] Ball, P. Nature's patterns, Oxford: Oxford University Press.(2011) 